\title{
NUMERICAL RANGES OF RESTRICTED SHIFTS AND UNITARY DILATIONS
}

\author{
Is Abelle Chalendar, PAMEla Gorkin And JonAthan R. PARTington
}

Abstract. This paper considers the restricted shift operator associated with an infinite Blaschke product, expressing the closure of its numerical range as the intersection of the closures of the numerical ranges of a parametrized family of unitary dilations (or, equivalently, unitary perturbations of a modified restricted shift). The techniques used are based on interpolation and Clark measures. The results generalize known theorems for numerical ranges of matrices associated with finite-dimensional Blaschke products, which can be expressed geometrically in terms of the Poncelet property.

Mathematics subject classification (2000): 30D50, 47A20, 15A60, 51N20.

Keywords and phrases: Blaschke product, restricted shift, numerical range, dilation, Poncelet property.

\section{REFERENCES}

[1] G. CASSIER AND I. CHALENDAR, The group of the invariants of a finite Blaschke product, Complex variables, 42 (2000), 193-206.

[2] I. Chalendar, N. Chevrot And J.R. Partington, Nearly invariant subspaces for backward shifts on vector-valued Hardy spaces, Journal of Operator Theory, to appear.

[3] J.A. Cima, A.L. Matheson, And W.T. Ross, The Cauchy transform, Mathematical Surveys and Monographs, 125. American Mathematical Society, Providence, RI, 2006.

[4] U. Daepp, P. Gorkin And R. Mortini, Ellipses and finite Blaschke products, Amer. Math. Monthly, 109, 9 (2002), 785-795.

[5] U. DAEPP, P. GORKIN AND K. VOSS, What Blaschke products tell us about the numerical range and Poncelet curves, Preprint.

[6] E. B. DAVIES, Linear operators and their spectra, Cambridge University Press, 2007.

[7] S.R. GARCIA, Conjugation and Clark operators, in Recent advances in operator-related function theory, volume 393 of Contemp. Math., pages 67-111, Amer. Math. Soc., Providence, RI, 2006.

[8] J.B. Garnett, Bounded analytic functions. Academic Press, New York, 1981.

[9] H.-L. GAU AND P.Y. WU, Numerical range of $S(\phi)$, Linear and Multilinear Algebra, 45, 1 (1998), 49-73.

[10] H.-L. GAu And P.Y. Wu, Numerical Range and Poncelet Property, Taiwanese Journal of Mathematics, 7, 2 (2003), 173-193.

[11] P. Gorkin AND R.C. RhoAdes, Boundary interpolation by finite Blaschke products, Constr. Approx., 27, 1 (2008), 75-98.

[12] P.R. Halmos, Normal dilations and extensions of operators, Summa Brasil. Math., 2 (1950), 125134.

[13] M. Marden, Geometry of Polynomials, American Mathematical Society, Mathematical Surveys and Monographs 3, 1949, Reprinted 2005.

[14] B. MiRmAn, UB-matrices and conditions for Poncelet polygon to be closed, Linear Algebra Appl., 360 (2003), 123-150.

[15] D. SARASON, Algebraic properties of truncated Toeplitz operators, Operators and Matrices, 1 (2007), 491-526.

[16] B. Sz.-NAgy, And C. FoIAŞ, Harmonic analysis of operators on Hilbert space, North-Holland Publishing Co., Amsterdam-London, 1970. 